openUP - February 2007

\title{
Structural and mechanistic insights into the action of Plasmodium falciparum spermidine synthase
}

\author{
Pieter B. Burger ${ }^{a}$, Lyn-Marie Birkholtz ${ }^{b}$, Fourie Joubert ${ }^{a}$, Nashya Haider ${ }^{c}$, Rolf D. \\ Walter $^{c}$ and Abraham I. Louw ${ }^{\text {b }}$ \\ ${ }^{a}$ Bioinformatics and Computational Biology Unit, Faculty of Natural and Agricultural \\ Sciences, University of Pretoria, Pretoria 0002, South Africa \\ ${ }^{\mathrm{b}}$ Department of Biochemistry, Faculty of Natural and Agricultural Sciences, University \\ of Pretoria, Pretoria 0002, South Africa \\ ${ }^{c}$ Biochemical Parasitology, Bernard Nocht Institute for Tropical Medicine, Hamburg, \\ Germany
}

\begin{abstract}
Spermidine synthase is currently considered as a promising drug target in the malaria parasite, Plasmodium falciparum, due to the vital role of spermidine in the activation of the eukaryotic translation initiation factor (eIF5A) and cell proliferation. However, very limited information was available regarding the structure and mechanism of action of the protein at the start of this study. Structural and mechanistic insights of the P. falciparum spermidine synthase (PfSpdSyn) were obtained utilizing molecular dynamics simulations of a homology model based on the crystal structures of the Arabidopsis thaliana and Thermotoga maritima homologues. Our data are supported by in vitro site-directed mutagenesis of essential residues as well as by a crystal structure of the protein that became available recently. We provide, for the first time, dynamic evidence for the mechanism of the aminopropyltransferase action of PfSpdSyn. This characterization of the structural and mechanistic properties of the PfSpdSyn as well as the elucidation of the active site residues involved in substrate, product, and inhibitor interactions paves the way toward inhibitor selection or design of parasite-specific inhibitors.
\end{abstract}




\section{Graphical abstract}

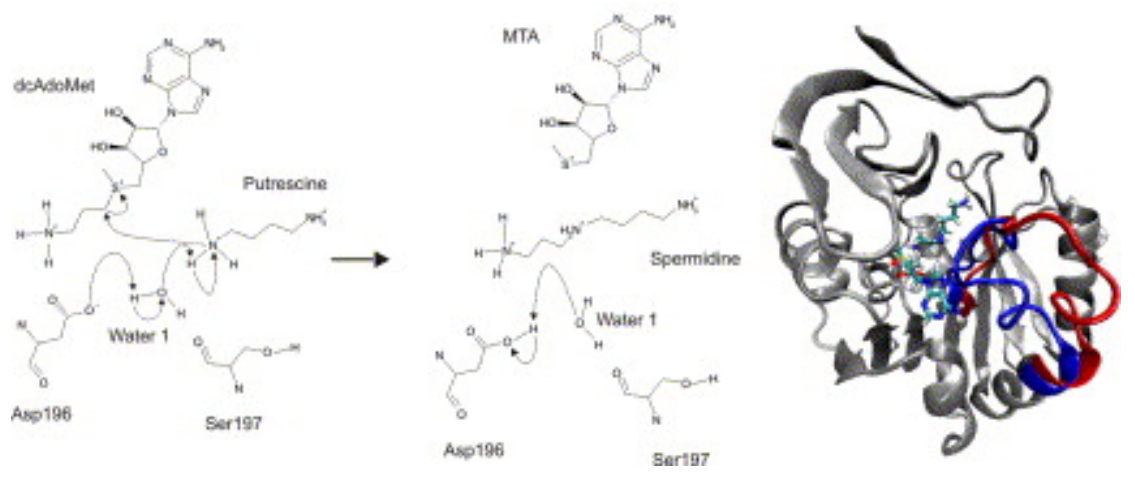

\section{Article Outline}

1. Introduction

2. Results and discussion

2.1. Comparative modeling of PfSpdSyn

2.2. Binding cavity analysis

2.3. Dynamic protein-substrate interaction analyses

2.4. Proposed mechanism of action of PfSpdSyn mediated by a gate-keeping loop

2.5. In vitro validation by site-directed mutagenesis

2.6. Inhibitor studies

3. Conclusion

4. Methods

4.1. Homology model construction

4.2. Binding site analysis

4.3. Protein-substrate interactions

4.4. Molecular dynamics

4.5. Validation of homology model by site-directed mutagenesis

4.6. Site-directed mutagenesis and functional analysis of recombinant PfSpdSyn Acknowledgements

References

\section{Introduction}

Malaria is an infectious disease that affects the daily lives of almost $40 \%$ of the world's population, causing 300-500 million clinical cases annually and resulting in approximately 2 million deaths. These deaths are mainly due to the most virulent malaria 
species, Plasmodium falciparum. ${ }^{1}$ The lack of a vaccine and the rapid emergence and spread of drug-resistant strains of $P$. falciparum necessitate the development of new drugs and the identification and validation of new parasite-specific therapeutic targets. One potential parasite metabolic target is polyamine biosynthesis.

Polyamines are essential and ubiquitous, aliphatic amines, which include putrescine, spermidine, and spermine. The biosynthesis of these polyamines peaks during cell proliferation and differentiation. ${ }^{2}$ Although the absolute molecular functions of polyamines are still unclear, they are thought to play an important role in the stabilization of DNA and RNA, phospholipids, and various proteins in vivo., ${ }^{3,4}$ and 5 Ornithine decarboxylase (ODC) catalyzes the decarboxylation of ornithine to produce putrescine, which serves as a scaffold for the addition of an aminopropyl group from $S$ adenosylmethionine (dcAdoMet), a product of $S$-adenosylmethionine decarboxylase (AdoMetDC) catalysis, to produce spermidine and spermine. The latter is catalyzed by spermidine and spermine synthase activities that produce 5'-methylthioadenosine (MTA) as byproduct of both reactions.

Polyamine metabolism has been extensively studied as a potential human cancer target with moderate success, since its inhibition generally results in cytostasis. ${ }^{6}$ This is thought to be mainly due to the short half-lives of the main regulatory enzymes, ODC and AdoMetDC. ${ }^{4 \text { and } 7}$ In contrast, inhibition of ODC by DL- $\alpha$-difluoromethylornithine (DFMO) is successfully used to treat African sleeping sickness caused by Trypanosoma brucei gambiense, thereby validating polyamine metabolism as an antiparasitic target in these protozoan parasites. ${ }^{8,9}$ and 10 DFMO is however not therapeutically used against $P$. falciparum, since its use is only cytostatic due to poor uptake of the drug and to exogenous putrescine uptake in these parasites. ${ }^{9}$

Studies directed at polyamine biosynthesis as a potential drug target in P. falciparum have mainly been focused on ODC and AdoMetDC with relatively little attention being paid to spermidine synthase (PfSpdSyn). Investigations of PfSpdSyn have been limited to molecular and biochemical characterizations including determination of the effects of specific inhibitors. ${ }^{11}$ Interestingly, this enzyme has several unique features including its ability to produce low levels of spermine found in the parasite, since no spermine synthase activity has been identified in P. falciparum. ${ }^{11}$ and 12 The essential nature of spermidine synthase in the parasite is reflected by the importance of its product, spermidine. In addition to the general stabilization of DNA and RNA by polyamines, it has been shown that spermidine increases DNA-polymerase activity in P. falciparum by sixfold. ${ }^{13}$ Spermidine is furthermore essential for the modification and activation of the eukaryotic translation initiation factor eIF5A and in trypanosomes for the biosynthesis of the glutathione mimic, trypanothione. ${ }^{14,15,16 \text { and } 17}$ Some effects of polyamine biosynthesis inhibitors have therefore been attributed to the accumulation of unmodified eIF5A due to spermidine depletion, which resulted, for example, in the suppression of multi-drugresistant human immunodeficiency virus type 1 (HIV-1) replication. ${ }^{13,18 \text { and } 19}$ Additionally, null-mutants of spermidine synthase showed that it is absolutely essential for the survival of other lower eukaryotes, including the protozoan Leishmania donovani. $^{20,21 \text { and } 22}$ 
The spermidine synthase inhibitor, dicyclohexylamine, has been shown to block $P$. falciparum growth by depleting the endogenous polyamine pool. ${ }^{23}$ More recently, trans4-methylcyclohexylamine (4MCHA) was also shown to be a potent inhibitor of PfSpdSyn. ${ }^{11}$ Treatment of P. falciparum cultures with $100 \mu \mathrm{M}$ 4MCHA leads to $85 \%$ growth arrest after $48 \mathrm{~h}$. The parasites could not be significantly rescued by the addition of exogenous spermidine, which was not the case with 3-aminooxy-1-aminopropane inhibition of ODC that could be reversed by addition of putrescine. ${ }^{11}$ and 24 However, the possibility of additional targets for the spermidine synthase inhibitors or inefficient uptake of spermidine could not be excluded. ${ }^{11}$

Also, although 4MCHA caused a significant reduction in the spermidine levels in rat hepatoma tissue cells (HTC), no reduction in cellular growth rate was observed due to compensatory increases in putrescine and spermine levels via the interconversion pathway. ${ }^{11,25}$ and 26 The apparent absence of a polyamine interconversion pathway in the malaria parasite coupled to the dependence of eIF5 on spermidine for activation marks PfSpdSyn as one of the most promising drug targets in the polyamine biosynthetic pathway and warrants further investigations into its structure-activity properties.

Spermidine synthases are part of the larger aminopropyltransferase family of proteins. A recent review by Ikeguchi et al. proposed a mechanism of catalysis via a $\mathrm{SN}_{2}$ reaction (Fig. 1) resulting in an inverse configuration of the methylene carbon undergoing nucleophilic attack by putrescine (via an attacking nitrogen), which is mediated by a gatekeeping loop. ${ }^{27,28}$ and 29 The majority of the spermidine synthase family members are homodimers, however in thermophiles these proteins occur as tetramers. PfSpdSyn is a dimer in solution with an approximate subunit molecular mass of $36.6 \mathrm{kDa} .{ }^{11}{ }^{\text {and } 27}$ It has significant sequence identity (49\%) with the spermidine synthase of Arabidopsis thaliana. ${ }^{11}$ Sequence identities of $>30 \%$ enable the creation of comparative or homology models of sufficient quality to be realistic in the depiction of protein-ligand interactions and to be useful in virtual screening and structure-based drug design. ${ }^{30}$

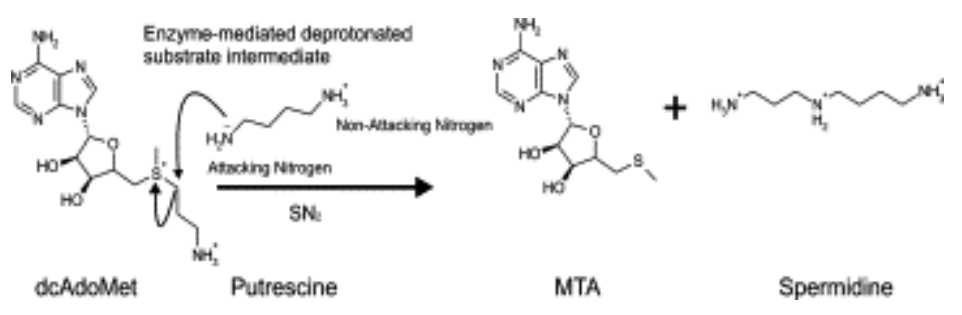

Figure 1. The current proposed mechanism of action for spermidine synthases. Enzymemediated deprotonation of the attacking nitrogen of putrescine results in a nucleophilic attack on an electrophilic carbon of dcAdoMet. This results in the formation of spermidine and MTA (Adapted from Ikeguchi et al.). ${ }^{27}$

This paper provides insights into the structural features and mechanistic details of PfSpdSyn based on molecular dynamics simulations of a homology model, which is 
supported by site-directed mutagenesis experiments. Special emphasis is placed on the elucidation of the protein-substrate interactions.

\section{Results and discussion}

\subsection{Comparative modeling of PfSpdSyn}

Comparative modeling uses experimentally determined structures to predict the conformation of proteins with similar sequences. This technique is based on the observation that in nature the structural conformation of a protein is more conserved than its amino acid sequence. ${ }^{31}$ Thirty-four spermidine synthase sequences were retrieved from the UniProt database, which were associated with four structures from PDB. ${ }^{32}$ and 33 The T-Coffee package was used to construct a protein family alignment to optimize the target-template alignment. ${ }^{34}$ The crystal structures of A. thaliana (AtSpdSyn) and Thermotoga maritima (TmSpdSyn) spermidine synthase were used as templates for modeling. The first 39 amino acids of the PfSpdSyn sequence were omitted due to insufficient template match (Fig. 2). AtSpdSyn was used due to its high sequence identity and that of TmSpdSyn since it contained the substrate analogue, $S$-adenosyl-1,8-diamino3-thiooctane (AdoDATO). Thus, AdoDATO is a compound containing both substrate and product moieties. ${ }^{29}$ AdoDATO will be referred to as a substrate analogue from here on. Models were built using Modeller with and without AdoDATO. ${ }^{35}$ The active site residues interacting with AdoDATO appear to be highly conserved between the human spermidine synthase (HsSpdSyn), TmSpdSyn, AtSpdSyn, and PfSpdSyn (Table 1). 


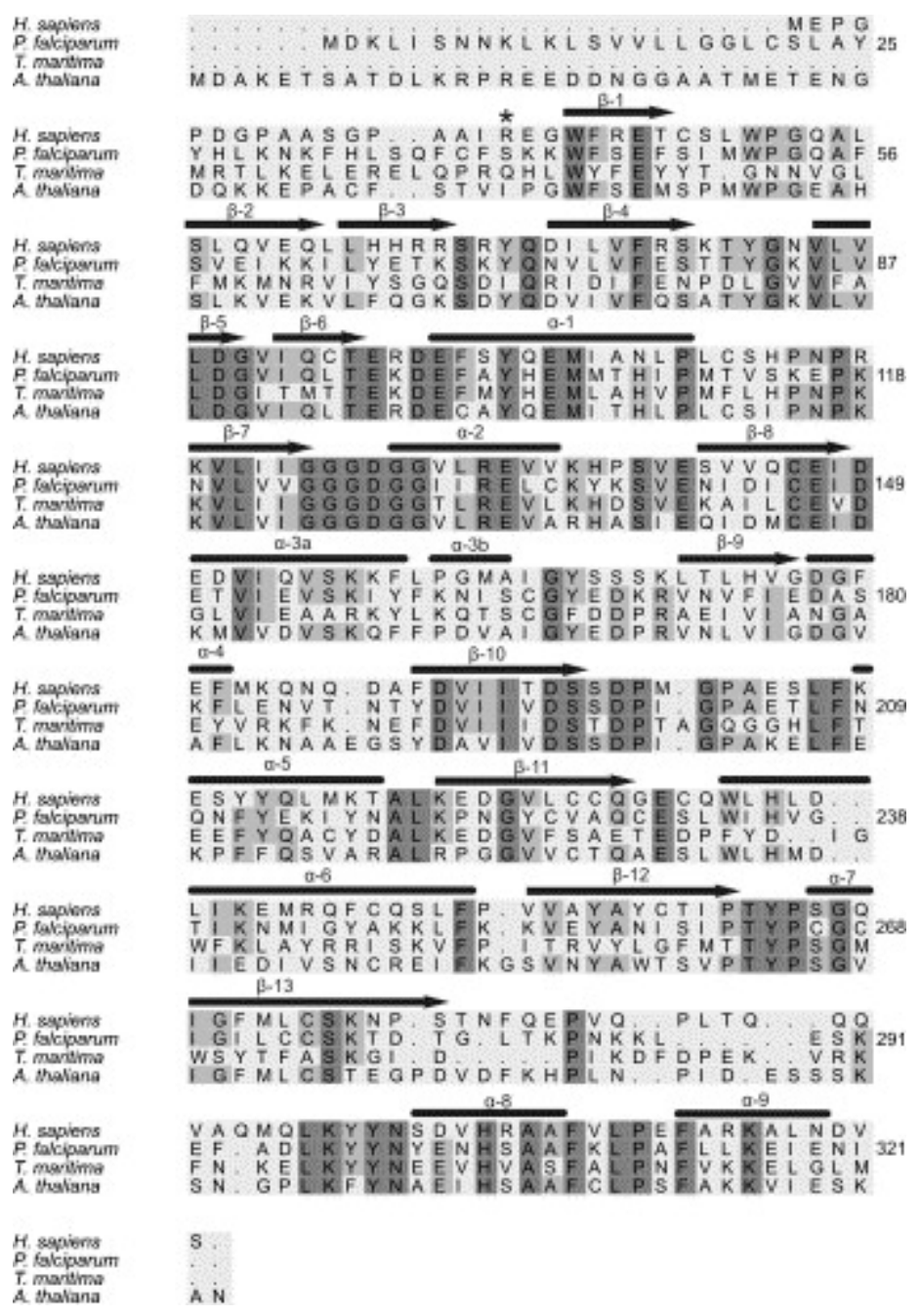

Figure 2. An alignment of the protein sequences of $P$. falciparum spermidine synthase and the spermidine synthases of $A$. thaliana and $T$. maritima used as templates during homology modeling. Also included in the alignment is the protein sequence of the human spermidine synthase. The cylinders indicate helices and the arrows indicate $\beta$-strands. The amino acids shaded light gray represent conservation between $50 \%$ and $80 \%$, whereas the dark gray areas represent residues with conservation higher than $80 \%$. The numbering used is in reference to the amino acid sequence of PfSpdSyn. ${ }^{*}$ indicates start of homology model. 
Table 1.

A comparison between the active site residues of Thermotoga maritima (TmSpdSyn), Homo sapiens (HsSpdSyn), Arabidopsis thaliana (AtSpdSyn) and Plasmodium falciparum (PfSpdSyn) spermidine synthases, indicating its conserved nature

\begin{tabular}{|c|c|c|c|}
\hline TmSpdSyn & HsSpdSyn & AtSpdSyn & PfSpdSyn \\
\hline $\mathrm{Gln}_{46}{ }^{\mathrm{a}}$ & $\mathrm{G} \ln _{49}$ & $G \ln _{76}$ & $\mathrm{G} \ln _{72}{ }^{\mathrm{a}}$ \\
\hline $\mathrm{Leu}_{62}$ & $\mathrm{Leu}_{65}$ & $\mathrm{Leu}_{92}$ & $\mathrm{Leu}_{88}$ \\
\hline $\mathrm{Met}_{67}$ & $\mathrm{Gln}_{170}$ & $\mathrm{Gln}_{97}$ & $\mathrm{Gln}_{93}$ \\
\hline $\mathrm{Tyr}_{76}$ & $\mathrm{Tyr}_{79}$ & $\mathrm{Tyr}_{106}$ & $\mathrm{Tyr}_{102}$ \\
\hline $\mathrm{His}_{77}{ }^{\mathrm{a}}$ & $\mathrm{Gln}_{80}$ & $\mathrm{Gln}_{107}$ & $\mathrm{His}_{103}{ }^{\mathrm{a}}$ \\
\hline Gly $_{98}$ & Gly $_{101}$ & Gly $_{128}$ & Gly $_{124}$ \\
\hline $\mathrm{Gly}_{99}$ & Gly $_{102}$ & Gly $_{129}$ & Gly $_{125}$ \\
\hline Asp $_{101}{ }^{a}$ & Asp $_{104}$ & Asp $_{131}$ & Asp $_{127}{ }^{a}$ \\
\hline $\mathrm{Glu}_{121}{ }^{\mathrm{a}}$ & $\mathrm{Glu}_{124}$ & $\mathrm{Glu}_{151}$ & $\mathrm{Glu}_{147^{\mathrm{a}}}$ \\
\hline $\mathrm{Val}_{122}$ & $\mathrm{Ile}_{125}$ & $\mathrm{Ile}_{152}$ & $\mathrm{Ile}_{148^{2}}$ \\
\hline $\mathrm{Gly}_{151}$ & Gly $_{154}$ & $\mathrm{Gly}_{181}$ & $\mathrm{Ala}_{179}{ }^{\mathrm{a}}$ \\
\hline $\mathrm{Asn}_{152}{ }^{\mathrm{a}}$ & Asp $_{155}$ & Asp 182 & Asp $_{178^{a}}$ \\
\hline Asp $_{170^{a}}{ }^{\mathrm{a}}$ & $\mathrm{Asp}_{173}$ & Asp $_{201}$ & Asp $196{ }^{a}$ \\
\hline $\operatorname{Ser}_{171}$ & $\operatorname{Ser}_{174}$ & Ser $_{202}$ & $\operatorname{Ser}_{197}$ \\
\hline $\mathrm{Thr}_{172}$ & $\operatorname{Ser}_{175}$ & $\mathrm{Ser}_{203}$ & $\operatorname{Ser}_{198}$ \\
\hline Asp $_{173^{a}}{ }^{a}$ & Asp $_{176}$ & Asp $_{204}$ & Asp $_{199}{ }^{2}$ \\
\hline $\mathrm{Gln}_{178}{ }^{\mathrm{a}}$ & Pro $_{180}$ & $\mathrm{PrO}_{208}$ & $\mathrm{PrO}_{203}$ \\
\hline $\mathrm{Leu}_{182}$ & $\mathrm{Leu}_{184}$ & $\mathrm{Leu}_{212}$ & $\mathrm{Leu}_{207}$ \\
\hline $\mathrm{Tyr}_{239}$ & $\mathrm{Tyr}_{241}$ & $\mathrm{Tyr}_{270}$ & $\mathrm{Tyr}_{264}$ \\
\hline $\operatorname{Trp}_{244}$ & $\mathrm{Ile}_{246}$ & $\mathrm{Ile}_{275}$ & $\mathrm{Ile}_{269}$ \\
\hline
\end{tabular}

Shaded areas indicate non-identical residues between the four organisms.

${ }^{a}$ Indicates residues forming hydrogen bonds with AdoDATO.

Spermidine synthases are members of the putrescine aminopropyltransferase family that generally consists of a small N-terminal domain and a large catalytic C-terminal domain (Rossmann-like fold). The N-terminal domain of the monomer of PfSpdSyn consists of a six-stranded $\beta$-sheet ( $\beta 1-6$ on Fig. 3a) and the C-terminal domain of a seven-stranded $\beta$ sheet flanked by nine $\alpha$-helices $(\beta 7-13, \alpha 1-9$, Figure 2 and Figure $3 a)$. A Ramachandran plot of the PfSpdSyn model showed $87 \%$ of its residues to be in the most favorable region (Fig. 3b), which was similar to the AtSpdSyn and TmSpdSyn structures used as templates. PROCHECK confirmed that all the parameters of the PfSpdSyn model were within normal ranges. ${ }^{36}$ 

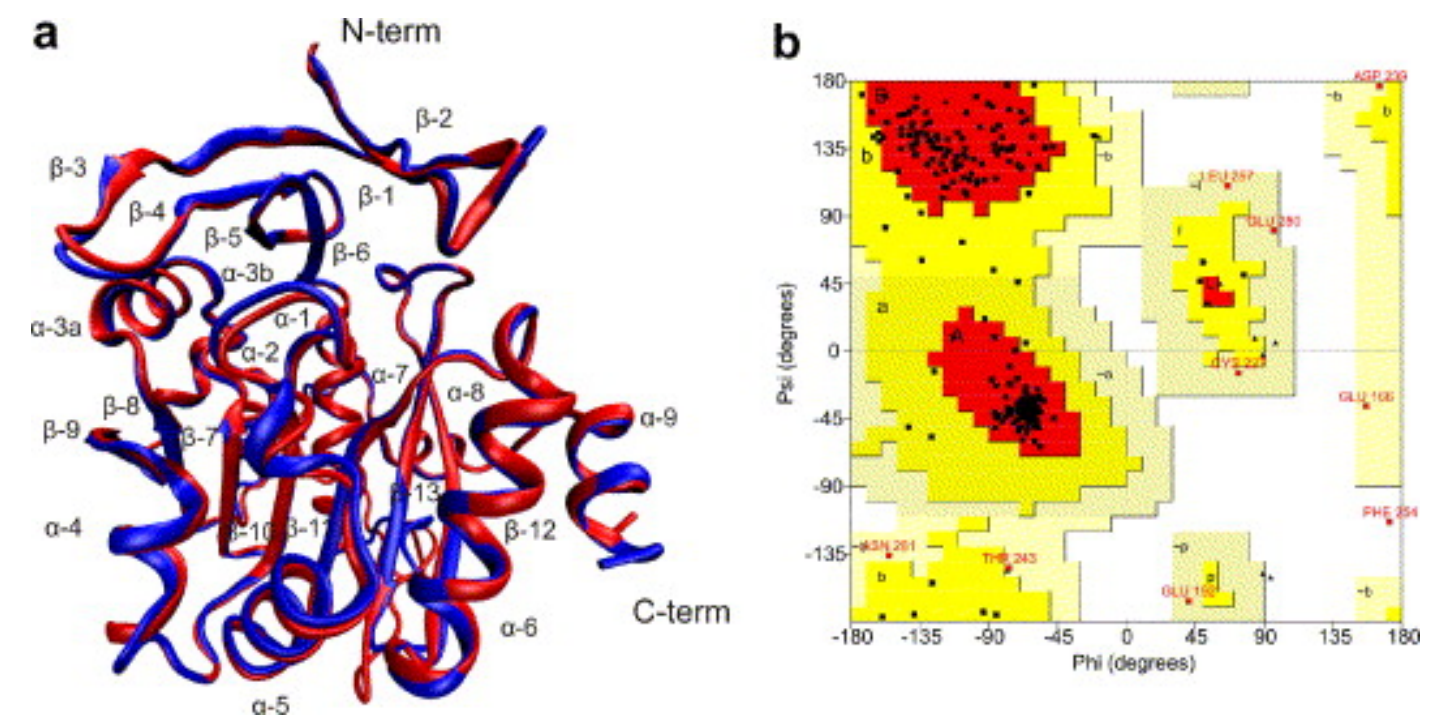

Figure 3. (a) A structural alignment of the PfSpdSyn model (red) and crystal structure (blue; PDB: entry 2HTE). (b) Ramachandran plot for the model of PfSpdSyn as produced by PROCHECK. Eighty-seven percent of the residues were within favorable structural areas.

While this paper was in preparation a crystal structure of PfSpdSyn (PDB entry: 2HTE) became available (http://sgc.utoronto.ca). Structural alignment revealed an excellent correlation between the $\alpha \mathrm{C}$-backbone of the crystal structure and model of the PfSpdSyn (RMSD $0.594 \AA$; Fig. 3a). The active site residues identified to interact with AdoDATO, as illustrated in Table 1, were aligned and showed to be highly conserved (RMSD $0.476 \AA$ ). The significant correlation between the crystal structure and homology model of PfSpdSyn provides support for the quality of the model used during this study.

\subsection{Binding cavity analysis}

From the PfSpdSyn model, the binding sites for putrescine and dcAdoMet were apparent (Fig. 4a). The dcAdoMet binding cavity is represented by the residues surrounding the adenosyl moiety of AdoDATO, whereas the residues surrounding the polyamine moiety represent the putrescine binding cavity. The putrescine binding cavity in PfSpdSyn has a central hydrophobic region flanked by two negatively charged regions in agreement with suggestions by Korolev et al. and Shirahata et al. ${ }^{25}$ and 29 This region is composed of Trp $\mathrm{p}_{51}$, $\mathrm{Val}_{91}, \mathrm{Tyr}_{102}, \mathrm{Ile}_{235}, \mathrm{Tyr}_{246}, \mathrm{Pro}_{247}$, and $\mathrm{Ile}_{269}$ (not shown). ${ }^{29}$ and 27 The two negatively charged/electron-donating regions consist of Gln $93, \mathrm{Tyr}_{102}, \mathrm{Asp}_{196}, \mathrm{Ser}_{197}, \mathrm{Gln}_{229}$, and $\mathrm{Glu}_{231}$, Asp $_{199}$, His $_{236}$, respectively (Fig. 4a). 

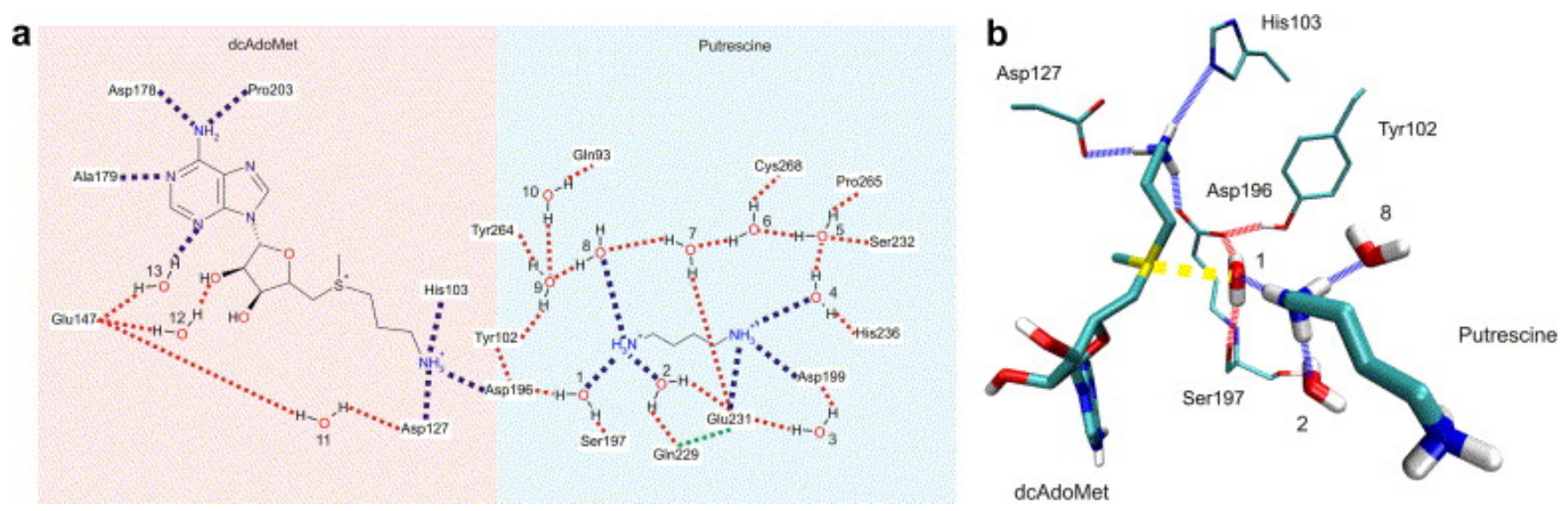

Figure 4. (a) A 2D representation of the interactions between PfSpdSyn and its substrates. The dcAdoMet binding cavity is represented in the apricot shaded area to the left and the putrescine binding cavity in the blue area to the right. Water molecules thought to anchor and orient putrescine (via hydrogen bonds represented as dashed red lines) are indicated and labeled numerically. Dashed blue lines indicate hydrogen bonds with nitrogen atoms. A protein-protein interacting hydrogen bond between $\mathrm{Gln}_{229}$ and $\mathrm{Glu}_{231}$ is represented in green. (b) A 3D representation of interactions showing the most important interactions for substrate binding and catalysis. Dashed lines in red and blue represent hydrogen bonds with the substrates. The polar interaction between water molecule 1 and the positively charged sulfur of dcAdoMet is colored in yellow.

AdoDATO was converted in silico to dcAdoMet and putrescine as an indirect means of determining protein-substrate interactions and the protein-substrate complex was subsequently minimized. Evaluation of the interactions between the substrates and PfSpdSyn revealed that $\mathrm{Tyr}_{102}, \mathrm{Asp}_{196}$, and $\mathrm{Ser}_{197}$ interact with the attacking nitrogen of putrescine. These residues have been suggested by Korolev et al. to play a central role in the deprotonation of putrescine. ${ }^{29}$ Interactions with dcAdoMet were conserved to those of the dcAdoMet moiety of AdoDATO (see below).

\subsection{Dynamic protein-substrate interaction analyses}

The PfSpdSyn model containing both substrates was subsequently solvated with water (TIP3) and subjected to molecular dynamic analysis to investigate the protein-substrate interactions in the presence of water. A simulation was performed with the attacking nitrogen of putrescine in the unprotonated state to ensure the correct orientation of the substrates in the protein. After about $20 \mathrm{ps,} \mathrm{a} \mathrm{protein} \mathrm{state} \mathrm{was} \mathrm{captured} \mathrm{and} \mathrm{the} \mathrm{attacking}$ nitrogen of putrescine protonated. The model was again subjected to molecular dynamics and a network of water molecules identified that potentially interact with the substrates (Fig. 4a). 
Eight hydrogen bonds were predicted between PfSpdSyn and dcAdoMet. Hydrogen bonds were directly formed between dcAdoMet and Asp 127, Asp $_{178}, A_{12} a_{179}$, Asp $_{196}$, His 103 , and Pro $_{203}$ (Fig. 4a). Glu 147 mediates two hydrogen bonds via water molecules 12 and 13, and an additional hydrogen bond with water molecule 11 , which in turn forms a hydrogen bond with $\mathrm{Asp}_{127}$. Asp $\mathrm{sp}_{127}$ together with His $\mathrm{Hi}_{103}$ and $\mathrm{Asp}_{196}$ forms hydrogen bonds with the aminopropyl chain of dcAdoMet (Fig. 4a and b). It is proposed that these three hydrogen bonds are necessary to orient the aminopropyl chain in order to present the electrophilic carbon for a nucleophilic attack by putrescine.

Only two direct hydrogen bonds are formed via residues Asp $\mathrm{Apg}_{199}$ and $\mathrm{Glu}_{231}$ between PfSpdSyn and putrescine. However, an extensive network of ten water molecules was found around putrescine, which is thought to play a role in anchoring and orienting putrescine in the active site in such a way that catalysis can take place (Fig. 4a). The attacking nitrogen forms hydrogen bonds with water molecules 1,2 , and 8 . The nonattacking nitrogen of putrescine forms three hydrogen bonds with water molecule 4, $\mathrm{Asp}_{199}$ and $\mathrm{Glu}_{231}$ (Fig. 4a and b).

Water molecule 1 was of most interest since it was anchored between $\mathrm{Asp}_{196}$ and the carbonyl group of $\operatorname{Ser}_{197}$ most of the time during the $1 \mathrm{~ns}$ simulation (Fig. 4b). It forms hydrogen bonds with $\mathrm{Asp}_{196}$ and $\mathrm{Ser}_{197}$, and is oriented in such a way that a hydrogen bond is formed with the attacking nitrogen of putrescine as well as a polar interaction with the positively charged sulfur of dcAdoMet. It therefore appears that water molecule 1 plays an important role during catalysis by bringing and holding together both the substrates. The importance of this water molecule was further supported by a $1 \mathrm{~ns}$ simulation of the PfSpdSyn model without substrates, which showed the presence of a water molecule between $\mathrm{Asp}_{196}$ and $\mathrm{Ser}_{197}$ throughout the simulation. Although this water molecule might get exchanged for another during the simulation, a water molecule was consistently found to occupy this molecular space. This water molecule also alternated its hydrogen bonds between $\mathrm{Asp}_{196}$, Ser $_{197}$, and $\mathrm{Gln}_{229}$ in the absence of substrates. Similar water molecules were also evident in the crystal structures of PfSpdSyn $(\mathrm{HOH} 8)$ and AtSpdSyn (HOH 125). The crystal structure of TmSpdSyn without AdoDATO (1INL) showed a similar water molecule as in the above mentioned structures in chain C only (HOH 687), whereas in the presence of AdoDATO (structure 1JQ3), no such water molecule was found. In the AdoDATO co-crystallized quaternary TmSpdSyn structure, one of the monomers has an unresolved gate-keeping loop and the other three have AdoDATO crystallized within it therefore not allowing the anchoring of the water molecule because the aminopentyl chain (putrescine moiety) of AdoDATO would displace it when AdoDATO binds.

Hydrogen bonds corresponding to the bonds formed between $\operatorname{Tyr}_{102}$ and $\mathrm{Asp}_{196}$ in the PfSpdSyn model are also conserved within the crystal structures of TmSpdSyn (without AdoDATO), PfSpdSyn, AtSpdSyn, and HsSpdSyn. It is proposed that the hydrogen bond formed between $\mathrm{Tyr}_{102}$ and $\mathrm{Asp}_{196}$ orients $\mathrm{Asp}_{196}$ in such a way that a hydrogen bond is formed with the aminopropyl chain of dcAdoMet, which together with $\mathrm{His}_{103}$ and $\mathrm{Asp}_{127}$ anchors this chain. This further supports the important role of $\mathrm{Asp}_{196}$ in the anchoring of 
the aminopropyl chain and the orientation of the electrophilic carbon, allowing for a nucleophilic attack by putrescine.

\subsection{Proposed mechanism of action of PfSpdSyn mediated by a gate-keeping loop}

Based on the results presented here, water molecule 1 (which anchors residues Asp 196 and Ser $_{197}$ ) is therefore proposed to play an important role during the aminopropyl transfer reaction by facilitating the deprotonation of putrescine to allow an electrophilic attack on dcAdoMet (Fig. 5a). It is possible that $\mathrm{Asp}_{196}$ removes a hydrogen from water molecule 1 , which in turn deprotonates the attacking nitrogen of putrescine. The deprotonated nitrogen consequently attacks the electrophilic carbon of dcAdoMet resulting in the addition of the aminopropyl chain to putrescine. This then results in the formation of the two products, spermidine and MTA. After the formation of the products, water molecule 1 removes the hydrogen from Asp $_{196}$ releasing it into the system and by so doing, regenerates the normal state of the protein. Further analysis is currently being performed to confirm this involvement of the water molecule.

a

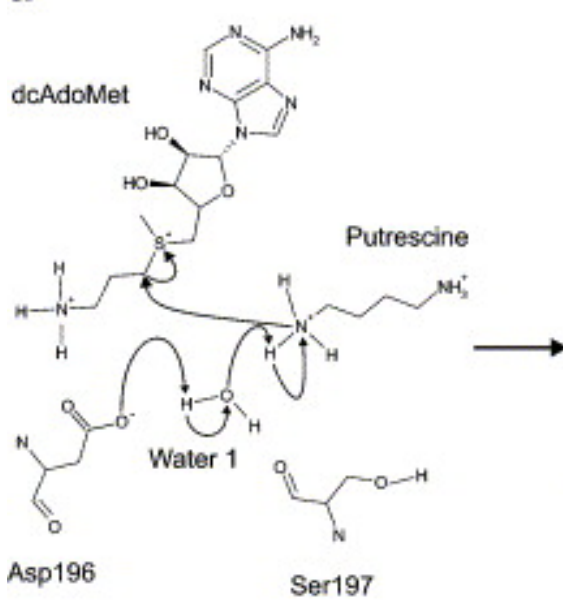

MTA

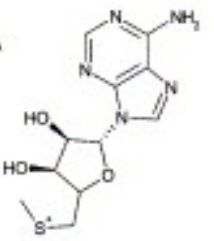

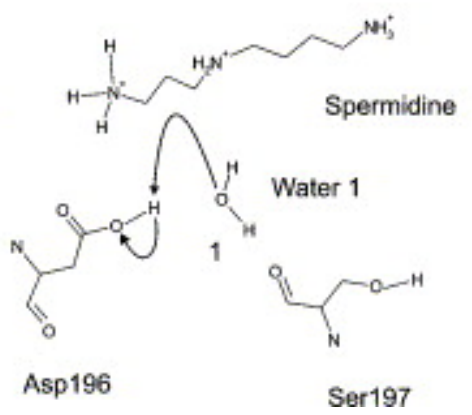

b

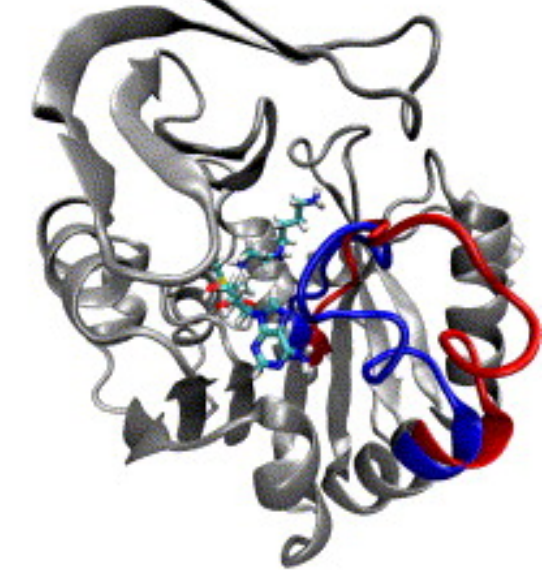

Figure 5. (a) A more detailed mechanism of action is presented for PfSpdSyn. Water molecule 1 is attacked by Asp 196 removing a hydrogen from it. The attacking nitrogen of putrescine is in turn deprotonated by the water molecule to enable a nucleophilic attack on the electrophilic carbon of dcAdoMet and the formation of spermidine and MTA. Asp $_{196}$ is deprotonated by water molecule 1 in order to regenerate the active form of PfSpdSyn for further catalysis to take place. (b) Loop movement reported during molecular dynamics of the PfSpdSyn containing the products MTA and spermidine. The gate-keeping loop of PfSpdSyn in both an open (red) and closed conformation (blue) is represented by a cartoon. 
Aminopropyltransferase reactions are proposed to be mediated by a gate-keeping loop. ${ }^{27}$ and 29 The PfSpdSyn model containing both reaction products (spermidine and MTA) was created and evaluated with molecular dynamics. It was observed that during this simulation, the gate-keeping loop covering entry to the active site is mobile and opens up (Fig. 5b). Subsequently, MTA started to move out of the active site as the gate-keeping loop opens after which spermidine can exit. Therefore, this dynamic visualization of the gate-keeping loop mobility provides for the first time evidence of gatekeeper activity in the spermidine synthase active site.

\subsection{In vitro validation by site-directed mutagenesis}

Site-directed mutagenesis was utilized to test the proposed importance of residues $\operatorname{Tyr}_{102}$, Asp $_{196}$, and $\operatorname{Ser}_{197}$. Tyr ${ }_{102}$ and Ser $_{197}$ were mutated to Ala, and Asp 196 was mutated to Asn. The Asp ${ }_{196}$ Asn mutation showed an $89 \%$ loss of activity, the $\mathrm{Tyr}_{102}$ Ala change resulted in $91 \%$ loss of activity, whereas the Ser ${ }_{197}$ Ala mutant had only $24 \%$ loss in activity (Fig. 6a). These mutant forms of the protein were also analyzed in silico on the PfSpdSyn model that contained both the natural substrates.

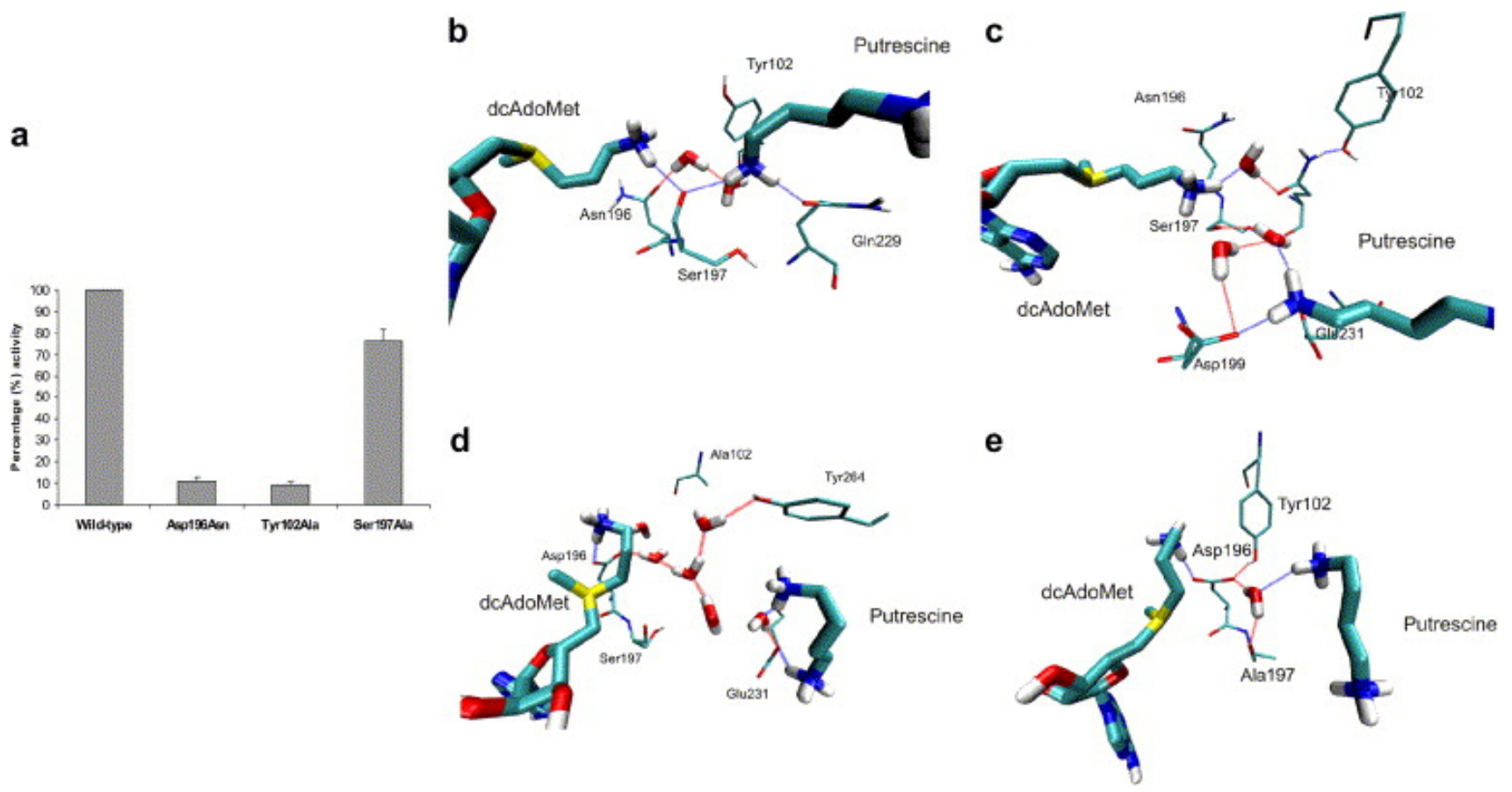

Figure 6. Graphical presentation of specific activity after point mutations performed on PfSpdSyn. (a) Effect of the point mutations Asp ${ }_{196}$ Asn, Ser ${ }_{197} A l a$, and $\operatorname{Tyr}_{102}$ Ala on the specific activity of PfSpdSyn compared to the wild-type. (b) The Asp ${ }_{196}$ Asn point mutation with the amine group of Asn pointing into the aminopropyl binding cavity (Scenario 1). (c) The Asp ${ }_{196}$ Asn point mutation with the carbonyl oxygen of Asn pointing into the aminopropyl binding cavity (Scenario 2). (d) Point mutation $\mathrm{Tyr}_{102} \mathrm{Ala}$ with the most important hydrogen bond formations indicated. (e) Point mutation Ser ${ }_{197}$ Ala with the most important hydrogen bond formations indicated. 
As shown above (Fig. 4), one of the oxygens of Asp $_{196}$ protrudes into the aminopropyl binding cavity, anchoring the aminopropyl group of dcAdoMet, whereas the other is oriented toward the putrescine binding cavity. Structural analysis of the Asp ${ }_{196} \mathrm{Asn}$ mutant PfSpdSyn model evaluated by molecular dynamics indicated that the replacement of the carboxylic acid of Asp by the amide group of Asn results in two different orientations of the amide group (Fig. $6 \mathrm{~b}$ and c). In the first case, the nitrogen of the amide group protrudes into the aminopropyl binding cavity and in the second case it is oriented toward the putrescine binding cavity. Simulation of the first scenario suggested that the anchoring of the aminopropyl chain of dcAdoMet was weakened by the loss of its hydrogen bond with $\mathrm{Asp}_{196}$ and it consequently moved out of its binding pocket into the catalytic space. This in turn caused putrescine to move deeper into its binding cavity with the attacking nitrogen of putrescine now interacting with $\mathrm{Gln}_{229}$ and the backbone oxygen of $\operatorname{Ser}_{197}$ (Fig. 6b). The hydrogen bond between $\mathrm{Asp}_{196}$ and $\mathrm{Tyr}_{102}$ is also lost after the introduction of the mutation. It is therefore neither possible for the protein to anchor the aminopropyl chain of dcAdoMet nor to anchor the attacking nitrogen of putrescine, which explains the drastic reduction in catalysis. In the second scenario, a hydrogen bond with $\mathrm{Tyr}_{102}$ is not formed resulting in the rotation of the amide group of $\mathrm{Asn}_{196}$. This allowed the aminopropyl group of dcAdoMet to move out of its binding pocket and consequently also out of the catalytic center of the protein. Putrescine subsequently moved deeper into its binding cavity with its attacking nitrogen now forming hydrogen bonds with $\mathrm{Glu}_{231}$, Asp 199 , and a water molecule mediated by the backbone oxygen of $\operatorname{Ser}_{197}$ (Fig. 6c). Both these simulated scenarios indicated that the Asp ${ }_{196}$ Asn mutation distorted the anchoring and orientation of putrescine and the aminopropyl chain of dcAdoMet and hence caused the marked (89\%) reduction in activity.

Simulation of the $\mathrm{Tyr}_{102}$ Ala mutation on the PfSpdSyn model indicated that this mutation also drastically altered the environment of the active site, inducing a loss of the hydrogen bond between $\operatorname{Tyr}_{102}$ and $\mathrm{Asp}_{196}$ (Fig. 6d). The simulation indicated that this hydrogen bond loss allowed Asp $\mathrm{As}_{196}$ to move slightly toward the aminopropyl binding cavity of dcAdoMet, preventing the anchoring of a water molecule between Asp $_{196}$ and Ser $_{197}$. This in turn prevented the anchoring of the attacking nitrogen of putrescine. The attacking nitrogen of putrescine initially formed a novel hydrogen bond with a water molecule mediated by $\mathrm{Gln}_{93}$, which was later replaced by hydrogen bonds formed randomly with water molecules occupying the molecular space between putrescine and dcAdoMet (Fig. $6 \mathrm{~d})$. The hydrogen bond formation of the non-attacking nitrogen of putrescine was unchanged compared to the wild-type. The hydrogen bonds formed with the aminopropyl chain of dcAdoMet, Asp $_{127}$, Asp $_{196}$, and His 103 were retained. Since Asp A $_{196}$ was not anchored by the hydrogen bond formed with $\mathrm{Tyr}_{102}$ it was allowed to move, causing a shift in the orientation of the aminopropyl chain of dcAdoMet, which resulted in an unfavorable orientation between dcAdoMet and putrescine. The importance of $\mathrm{Tyr}_{102}$ in anchoring $\mathrm{Asp}_{196}$ for the proper orientation of both the aminopropyl chain of dcAdoMet and the attacking nitrogen is therefore reflected in the $91 \%$ loss in activity. 
The Ser $_{197}$ Ala mutation resulted in a $24 \%$ loss in activity. Ser ${ }_{197}$ together with Asp ${ }_{196}$ is thought to be important since they anchor water molecule 1 which in turn interacts with the attacking nitrogen of putrescine and the positively charged sulfur of dcAdoMet. During the simulation of the PfSpdSyn model containing the substrates, it was shown that water molecule 1 interacts with the backbone carbonyl group of $\operatorname{Ser}_{197}$, which was also true for the molecular dynamics simulation without any substrates (Fig. 4). It is therefore proposed that the interaction formed with the carbonyl group of Ala instead of the carbonyl group of Ser 197 with water molecule 1 allowed catalysis to take place only $76 \%$ of the time (Fig. 6e). The main change induced in the chemical environment of the active site was the loss of a hydrogen bond formed between $\operatorname{Ser}_{197}$ and $\mathrm{Gln}_{229}$, thought to give rigidity to the gate-keeping loop. The $\mathrm{Ser}_{197}$ Ala mutation is expected to make the loop more flexible and thus reducing the activity of the enzyme. Since this study was not intended to investigate loop movement, further analysis is however needed to confirm this hypothesis. As with the simulation of PfSpdSyn, containing both the substrates, a water molecule was identified during the PfSpdSyn Ser $_{197}$ Ala mutation simulation to play a role in the anchoring and orientation of both putrescine and the aminopropyl chain of dcAdoMet (Fig. 6e).

In summary, the mutation and molecular dynamic studies conclude that $\mathrm{Tyr}_{102}, \mathrm{Asp}_{196}$, and Ser $_{197}$ play important roles in catalysis of PfSpdSyn. Tyr ${ }_{102}$ is essential in the orientation of $\mathrm{Asp}_{196}$ to allow this residue to anchor both the aminopropyl chain of dcAdoMet and putrescine during catalysis. Ser ${ }_{197}$ was found to interact with its carbonyl backbone with water molecule 1 to facilitate the anchoring of putrescine. These data therefore validate the proposed mechanism of catalysis as predicted by the dynamic visualization of aminopropyl transfer in the PfSpdSyn model.

\subsection{Inhibitor studies}

As mentioned previously, investigations of seven known SpdSyn inhibitors have shown 4MCHA to be the most potent inhibitor of PfSpdSyn. ${ }^{11}$ This inhibitor was further investigated in this study to obtain a dynamic view of the aminopropyltransferase inhibition of PfSpdSyn using molecular dynamics. 4MCHA was overlaid with putrescine in its binding cavity and two different scenarios were simulated. The amine group of 4MCHA was aligned with either the attacking nitrogen of putrescine or the non-attacking nitrogen of putrescine. Both molecular dynamics simulations revealed that 4MCHA binds with its amine group in the putrescine binding cavity, partially filling it, whereas the hydrophobic moiety of the 4MCHA molecule protrudes into the adjacent hydrophobic cavity constituted by residues Met ${ }_{50}, \mathrm{Trp}_{51}, \mathrm{Phe}_{56}, \mathrm{Val}_{91}, \mathrm{Ile}_{92}, \mathrm{Tyr}_{102}, \mathrm{Ile}_{201}, \mathrm{Ile}_{235}, \mathrm{Tyr}_{264}$, and $\mathrm{Pro}_{265}$ (Fig. 7). This binding pattern correlates with previous reports, which proposed that the related inhibitor, cyclohexylamine, binds partially in the putrescine binding

cavity and the adjacent hydrophobic cavity. ${ }^{25,27,37}$ and 38 Since the only difference between cyclohexylamine and 4MCHA is the addition of a methyl group to 4MCHA, the PfSpdSyn simulations containing 4MCHA can additionally be used as evidence to support the proposed binding of cyclohexylamine. 


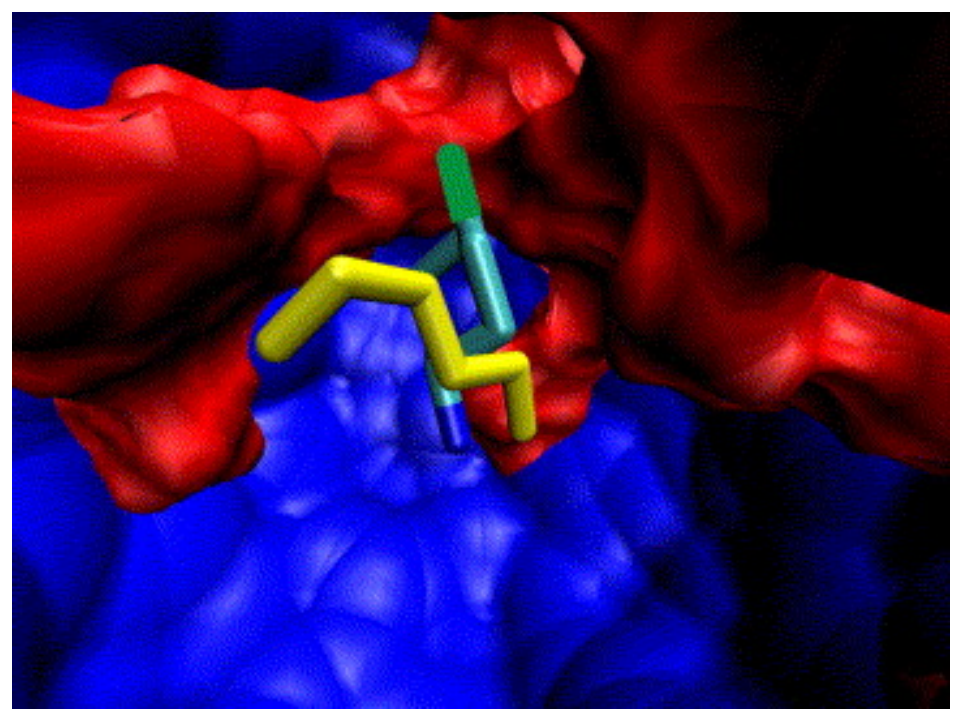

Figure 7. 4MCHA (cyan) was found to bind partially in the putrescine binding cavity with its nitrogen group (blue). The methylcyclohexyl group was found to bind in an adjacent hydrophobic cavity with the methyl group (green) lodged into the roof of the cavity. The hydrophobic regions are represented in red and the rest of the protein in blue. Putrescine is represented in yellow indicating the orientation of 4MCHA.

\section{Conclusion}

The analysis performed on the homology model of PfSpdSyn in this study is supported by an excellent correlation with the PfSpdSyn crystal structure, which became available during the preparation of this manuscript. We provide dynamic evidence of the mechanism of aminopropyltransferase action of PfSpdSyn. Essential residues were identified as $\mathrm{Tyr}_{102}, \mathrm{Asp}_{196}$, and $\mathrm{Ser}_{197}$ as well as a dynamic involvement of a water molecule. Molecular dynamic evaluations were supported by in vitro experimental data and indicated that $\mathrm{Tyr}_{102}$ is essential in the orientation of $\mathrm{Asp}_{196}$ to allow this residue to anchor both the aminopropyl chain of dcAdoMet and putrescine during catalysis. Ser $_{197}$ was found to interact with its carbonyl backbone with water molecule 1 to facilitate the anchoring of putrescine. Water molecule 1 facilitates the deprotonation of putrescine to allow an electrophilic attack on dcAdoMet. Finally, the aminopropyltransferase reaction is mediated by mobility of a gate-keeping loop to allow product release.

Drug discovery is a complex and expensive process, which is only one of the reasons for the limited availability of still-effective drugs against infectious diseases, especially malaria, which are prevalent in developing countries. Structure-based design methods coupled to bioinformatics applications have been instrumental in improving the efficiency to discover and optimize drug leads. ${ }^{39}$ The $3 \mathrm{D}$ description of the structural and mechanistic properties as well as functional groups involved in substrate, product, and inhibitor interactions of PfSpdSyn are essential components for the identification and 
development of parasite-specific inhibitors by structure-guided design and computational methods.

\section{Methods}

\subsection{Homology model construction}

Two crystal structures were used to construct the PfSpdSyn, which included the spermidine synthase crystal structures of T. maritima (1.80 $\AA$ resolution, PDB entry: 1JQ3) and A. thaliana (2.70 $\AA$ resolution, PDB entry: $1 \mathrm{XJ} 5)$. The spermidine synthase crystal structure from Caenorhabditis elegans has a sequence identity of $44 \%$ with PfSpdSyn but was omitted as a template since it did not contain a ligand and some loops were not resolved. ${ }^{40}$ The spermidine synthase crystal structure from T. maritima (TmSpdSyn; 1JQ3) was co-crystallized with a combined substrate-product analogue, Sadenosyl-1,8-diamino-3-thiooctane (AdoDATO). ${ }^{29}$ In an attempt to find an optimal alignment between the template and target sequences, the T-Coffee package was used to align 34 SpdSyn sequences retrieved from the UniProt and PDB databases, resulting in a protein family alignment. ${ }^{32,33 \text { and } 34}$ The experimentally aligned protein sequences of the spermidine synthases of T. maritima and A. thaliana were used as templates to construct the homology model of $P$. falciparum (Fig. 2). PfSpdSyn (UniProt entry: Q9FS5) was indicated to have a sequence identity of $32 \%$ and $49 \%$ with T. maritima and A. thaliana, respectively. Homology models of PfSpdSyn containing the substrate analogue, AdoDATO, were constructed using MODELLER 6v3 ${ }^{35}$ The PfSpdSyn models were subjected to stereochemical analysis, using PROCHECK, to evaluate the quality of the model. ${ }^{36}$

The PfSpdSyn homology models were further subjected to refinement by using the CHARMM (Chemistry at HARvard Molecular Mechanics) package. ${ }^{41}$ The partial charges used in the construction of the residue topology file of AdoDATO were computed using the MOPAC module within the InsightII (Accelrys) package. The PfSpdSyn models containing AdoDATO were subjected to 500 steps of steepest descent minimization followed by 50 steps of Adopted Basis Newton-Raphson (ABNR) minimization using the CHARMM27 all-atom empirical force field for proteins and nucleic acids. ${ }^{3}$ The protein-ligand interactions between the PfSpdSyn and TmSpdSyn structures were then compared using LIGPLOT. ${ }^{42}$

\subsection{Binding site analysis}

AdoDATO was removed from the PfSpdSyn model and docked back into the model using Cerius2 (Accelrys). The docked and build-in AdoDATO were compared with AdoDATO crystallized within TmSpdSyn. The comparison was made using LIGPLOT and visual inspection. ${ }^{42}$ The homology model of PfSpdSyn containing the build-in AdoDATO was used in further analysis. Evaluation of the binding cavity of PfSpdSyn was done using the LigandFit module of Cerius2 (Accelrys). Two binding cavities could be distinguished, one for binding of dcAdoMet and the other for putrescine binding. 


\subsection{Protein-substrate interactions}

Information obtained from the binding site analysis was subsequently used to elucidate protein-substrate interactions. The moieties of the substrate analogue AdoDATO constituting the substrates were identified. AdoDATO was subsequently converted into dcAdoMet and putrescine using InsightII (Accelrys). The attacking nitrogen of putrescine was built to be in the deprotonated state, since it needs to perform a nucleophilic attack on the electrophilic carbon of dcAdoMet. The PfSpdSyn model containing the newly formed substrates was then subjected to 100 steps of steepest descent minimization using CHARMM.$^{41}$ Putrescine adopted a strongly angular conformation as an artifact of the minimization conditions and was restored to a linear conformation using InsightII (Accelrys). The PfSpdSyn, AdoDATO complex was then further minimized for 400 steps using steepest descent minimization. To determine the protein-ligand interactions between PfSpdSyn and the protonated putrescine, a further 100 minimization steps were performed with putrescine in the protonated state. Protein-substrate interactions were evaluated using LIGPLOT and visual inspection. ${ }^{42}$

\subsection{Molecular dynamics}

Molecular dynamics was performed on the homology models containing the substrate analogue AdoDATO as well as the substrates, putrescine and dcAdoMet. The protein was solvated with TIP 3 water molecules. Molecular dynamics was started by 5000 steps of steepest descent minimization followed by 200 steps of ABNR minimization. The system was then heated to $310 \mathrm{~K}$ in steps of $5 \mathrm{~K}$ every 100 steps and left to equilibrate for $10 \mathrm{ps}$. The molecular dynamics simulation was subsequently performed for $1 \mathrm{~ns}$. VMD was used to visually inspect the molecular dynamics simulations of the homology models. ${ }^{43}$ The site-directed mutagenesis models were also subjected to molecular dynamics and the same procedure as above was followed. Molecular dynamics simulations were also performed on models containing the products, spermidine and MTA, as well as the SpdSyn inhibitor 4MCHA under the same conditions.

\subsection{Validation of homology model by site-directed mutagenesis}

Three in silico mutants were generated for the PfSpdSyn: Tyr $_{102}$ Ala, Asp ${ }_{196} A s n$, and $\mathrm{Ser}_{197}$ Ala. All mutants were constructed using the Biopolymer module in InsightII (Accelrys). The mutations were generated from PfSpdSyn models that contained both the natural substrates dcAdoMet and putrescine. The models were then subjected to molecular dynamics as described above.

\subsection{Site-directed mutagenesis and functional analysis of recombinant PfSpdSyn}

The wild-type expression construct pTRCHisB:PfSPDS2 as described in Haider et al. ${ }^{11}$ was used as template in subsequent site-directed mutagenesis experiments. Mutations were created to change residues $\mathrm{Tyr}_{102}$ and $\mathrm{Ser}_{197}$ to Ala and $\mathrm{Asp}_{196}$ to Asn. Primers used in the mutagenesis reactions were as follows $\left(5^{\prime}-3^{\prime}\right)$ : 
Asp ${ }_{196}$ Asn-sense: TATGATGTTATTATCGTAAATAGTTCAGATCCAATAGGA;

Asp ${ }_{196}$ Asn-antisense: TCCTATTGGATCTGAACTATTTACGATAATAACATCATA;

Ser ${ }_{197}$ Ala-sense: GATGTTATTATCGTAGATGCTTCAGATCCAATAGGACCA;

Ser ${ }_{197}$ Ala-antisense: TGGTCCTATTGGATCTGAAGCATCTACGATAATAACATC;

Tyr $_{102}$ Ala-sense: GAAAAAGATGAATTTGCTGCTCATGAAATGATGACACAT;

Tyr ${ }_{102}$ Ala-antisense: ATGTGTCATCATTTCATGAGCAGCAAATTCATCTTTTTC

Mutagenesis protocols were performed according to the methods as described previously. ${ }^{44}$ and ${ }^{45}$ Subsequently, wild-type as well as the three mutant proteins were expressed as His-tag fusion proteins in BLR(DE3) Escherichia coli as described by Haider et al. ${ }^{11}$ Proteins were isolated by Ni-affinity chromatography and aminopropyltransferase activities were determined by measuring the formation of ${ }^{14} \mathrm{C}$ labeled reaction products from 1,4 ${ }^{14} \mathrm{C}$-putrescine. ${ }^{11}$ Enzyme activities are expressed as specific activity $\left(\mathrm{nmol}^{-1} \mathrm{~min}^{-1}\right.$ mol of protein $\left.{ }^{-1}\right)$ and the results are means $\pm \mathrm{SD}$ of three independent experiments performed in duplicate with the specific activities normalized in reference to the appropriate controls for comparative purposes.

\section{References and notes}

1 J.G. Breman, Am. J. Trop. Med. Hyg. 64 (2001), p. 1.

2 C.A. Panagiotidis, S. Artandi, K. Calame and S.J. Silverstein, Nucleic Acids Res. 23 (1995), p. 1800.

3 K. Igarashi, I. Sakamoto, N. Goto, K. Kashiwagi, R. Honma and S. Hirose, Arch. Biochem. Biophys. 219 (1982), p. 438.

4 C.W. Tabor and H. Tabor, Adv. Enzymol. Relat. Areas Mol. Biol. 56 (1994), p. 251.

5 H.M. Wallace, A.V. Fraser and A. Hughes, Biochem. J. 376 (2003), p. 1.

6 L.J. Marton and A.E. Pegg, Annu. Rev. Pharmacol. Toxicol 35 (1995), p. 55.

7 J.E. Seely, H. Poso and A.E. Pegg, Biochem. J. 206 (1982), p. 311.

8 C.C. Wang, Annu. Rev. Pharmacol. Toxicol. 35 (1995), p. 93. 
9 S. Müller, G.H. Coombs and R.D. Walter, Trends Parasitol. 17 (2001), p. 242.

10 C.J. Bacchi and N. Yarlett, Mini Rev. Med. Chem. (2002), p. 553.

11 N. Haider, M. Eschbach, S. de Souza Dias, T. Gilberger, R.D. Walter and K. Luersen, Mol. Biochem. Parasitol. 142 (2005), p. 224.

12 A.E. Pegg, R. Poulin and J.K. Coward, Int. J. Biochem. Cell Biol. 27 (1995), p. 425.

13 U. Bachrach and L.A. Abu-Elheiga, Eur. J. Biochem. 191 (1990), p. 633.

14 T.L. Byers, B. Ganem and A.E. Pegg, Biochem. J. 287 (1992), p. 717.

15 T.L. Byers, R.S. Wechter, R.H. Hu and A.E. Pegg, Biochem. J. 303 (1994), p. 89.

16 S. Müller, E. Liebau, R.D. Walter and R.L. Krauth-Siegel, Trends Parasitol. 19 (2003), p. 320.

17 A. Kaiser, A. Gottwald, W. Maier and H.M. Seitz, Parasitol. Res. 91 (2003), p. 508.

18 M.H. Park, E.C. Wolff and J.E. Folk, Biofactors 4 (1993), p. 95.

19 B. Schäfer, I. Hauber, A. Bunk, J. Heukeshoven, A. Düsedau, D. Bevec and J. Hauber, J. Infect. Dis. 194 (2006), p. 740.

20 K. Guo, W.T. Chang and P.C. Newell, Biochim. Biophys. Acta 1449 (1999), p. 211.

21 S.C. Roberts, Y. Jiang, A. Jardim, N.S. Carter, O. Heby and B. Ullman, Mol. Biochem. Parasitol. 115 (2001), p. 217.

22 Y. Jin, J.W. Bok, D.G. de Pena and N.P. Keller, Mol. Microbiol. 46 (2002), p. 801.

23 A. Kaiser, A. Gottwald, C. Wiersch, B. Lindethal, W. Maier and H.M. Seitz, Parasitol. Res. 87 (2001), p. 963.

24 R. Das Gupta, T. Krause-Ilhe, B. Bergmann, I.B. Müller, A.R. Khumotov, S. Müller, R.D. Walter and K. Luersen, Antimicrob. Agents Chemother. 49 (2005), p. 2857.

25 A. Shirahata, N. Takahashi, T. Beppu, H. Hosoda and K.. Samejima, Biochem. Pharmacol. 44 (1991), p. 205.

26 T. Beppu, A. Shirahata, N. Takahashi, H. Hosoda and K. Samejima, J. Biochem. (Tokyo) 177 (1995), p. 339.

27 Y. Ikeguchi, M.C. Bewley and A.E. Pegg, J. Biochem. (Tokyo) 139 (2006), p. 1. 
28 G.R. Orr, D.W. Danz, G. Pontoni, P.C. Prabharakan, S.J. Gould and J.K. Coward, J. Am. Chem. Soc. 110 (1988), p. 5791.

29 S. Korolev, Y. Ikeguchi, T. Skarina, S. Beasley, C. Arrowsmith, A. Edwards, A.

Joachimiak, A.E. Pegg and A. Savchenko, Nat. Struct. Biol. 9 (2002), p. 27.

30 A. Hillisch, L.F. Pineda and R. Hilgenfeld, Drug Discovery Today 9 (2004), p. 659.

31 A.M. Lesk and C. Chothia, Philos. Trans. R. Soc. London B Biol. Sci. 317 (1986), p. 345.

32 R. Apweiler, A. Bairoch, C.H. Wu, W.C. Barker, B. Boeckmann, S. Ferro, E. Gasteiger, H. Huang, R. Lopez, M. Magrane, M.J. Martin, D.A. Natale, C. O'Donovan, N. Redaschi and L.L. Yeh, Nucleic Acids Res. 32 (2004), p. 115.

33 H.M. Berman, J. Westbrook, Z. Feng, G. Gilliland, T.N. Bhat, H. Weissig, I.N. Shindyalov and P.E. Bourne, Nucleic Acids Res. 28 (2000), p. 235.

34 C. Notredame, D.G. Higgins and J. Heringa, J. Mol. Biol. 302 (2000), p. 205.

35 A. Sali and T.L. Blundell, J. Mol. Biol. 234 (1993), p. 779.

36 R.A. Laskowski, M.W. MacArthur, D.S. Moss and J.M. Thornton, J. Appl. Crystallogr. 26 (1993), p. 283.

37 A.E. Pegg, R. Poulin and J.K. Coward, J. Biochem. 27 (1995), p. 425.

38 H. Goda, T. Watanabe, N. Takeda, M. Kobayashi, M. Wada, H. Hosoda, A. Shirahata and K. Samejima, Biol. Pharm. Bull. 27 (2004), p. 1327.

39 T.L. Blundell, B.L. Sibanda, R.W. Montalvão, S. Brewerton, V. Chelliah, C.L. Worth, N.J. Harmer, O. Davies and D. Burke, Philos. Trans. R. Soc. London, B. Biol. Sci. 361 (2006), p. 413.

40 V.T. Dufe, K. Lüersen, M. Eschbach, N. Haider, T. Karlberg, R.D. Walter and S. AlKaradaghi, FEBS Lett. 579 (2005), p. 6037.

41 B.R. Brooks, R.E. Bruccoleri, B.D. Olafson, D.J. States, S. Swaminanthan and M. Karplus, J. Comp. Chem. 4 (1983), p. 187.

42 A.C. Wallace, R.A. Laskowski and J.M. Thornton, Protein Eng. 8 (1995), p. 127.

43 W. Humphrey, A. Dalke and K. Schulten, J. Mol. Graph. 14 (1996), p. 338.

44 L. Birkholtz, C. Wrenger, F. Joubert, G. Wells, R.D. Walter and A.I. Louw, Biochem. J. 377 (2004), p. 439. 